\title{
Pt-Au Triangular Nanoprisms with Strong Dipole Plasmon Resonance for Hydrogen Generation Studied by Single-Particle Spectroscopy
}

Zaizhu Lou, Mamoru Fujitsuka, and Tetsuro Majima*

The Institute of Scientific and Industrial Research (SANKEN), Osaka University, Mihogaoka 8-1, Ibaraki Osaka 567-0047, Japan

* Address correspondence to

E-mail: majima@sanken.osaka-u.ac.jp 


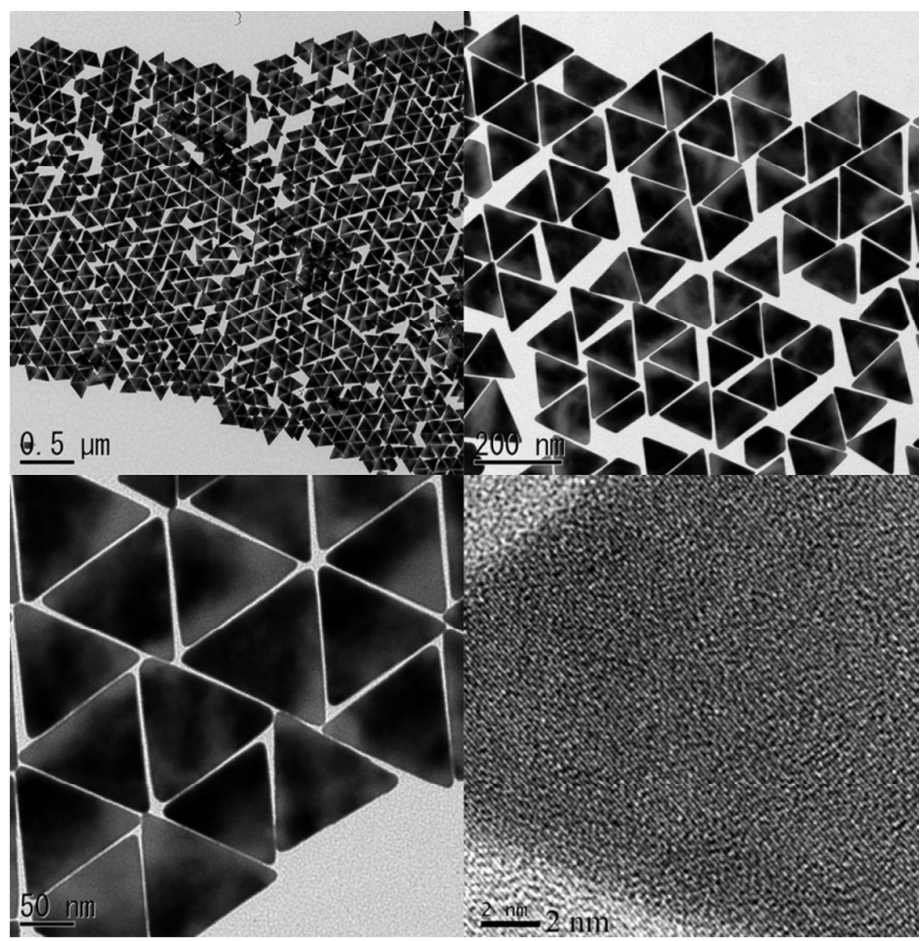

Figure S1. TEM images of Au TNPs.

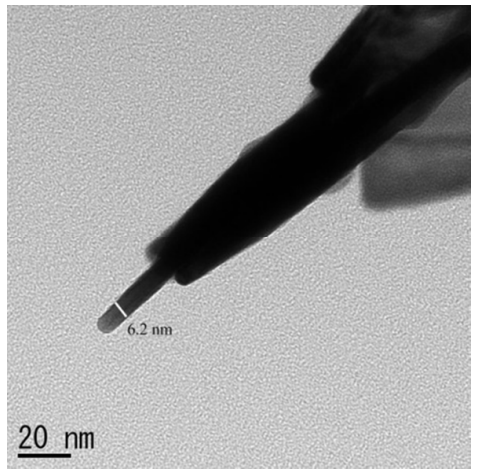

Figure S2. TEM image of Au TNPs with vertical view. The thickness of Au TNPs is about $6.2 \mathrm{~nm}$. 


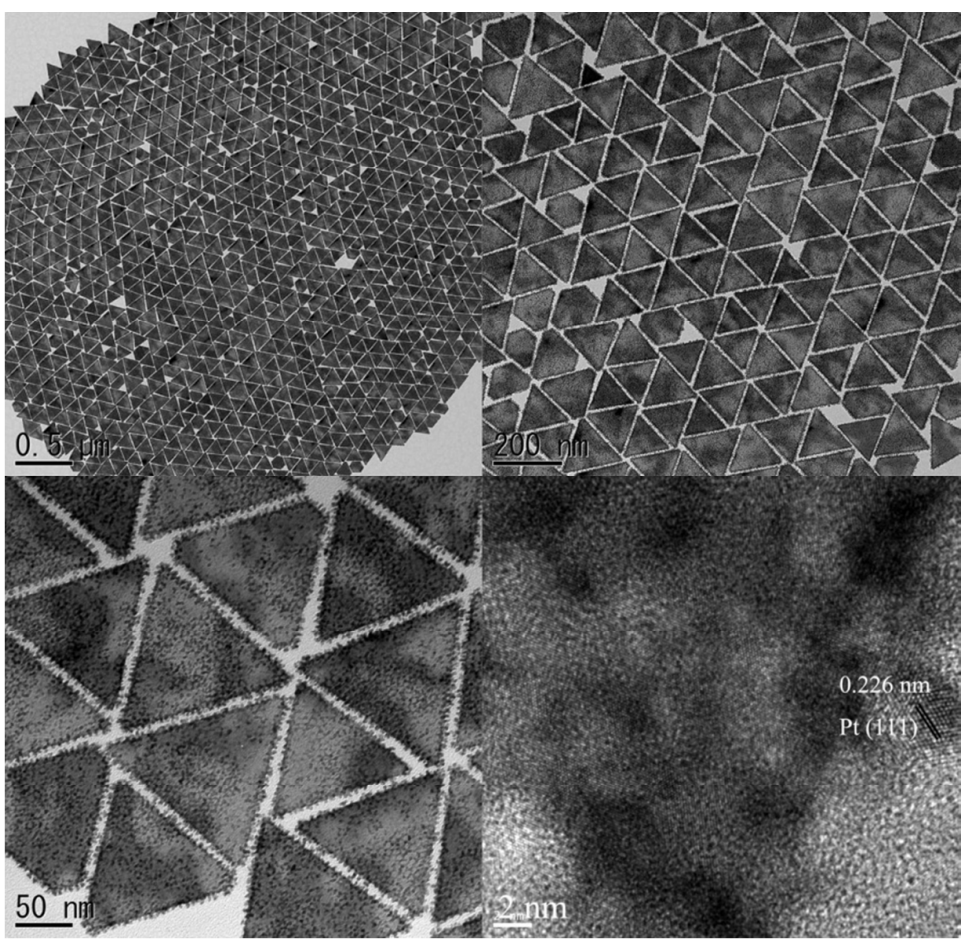

Figure S3. TEM images of Pt-covered Au TNPs.

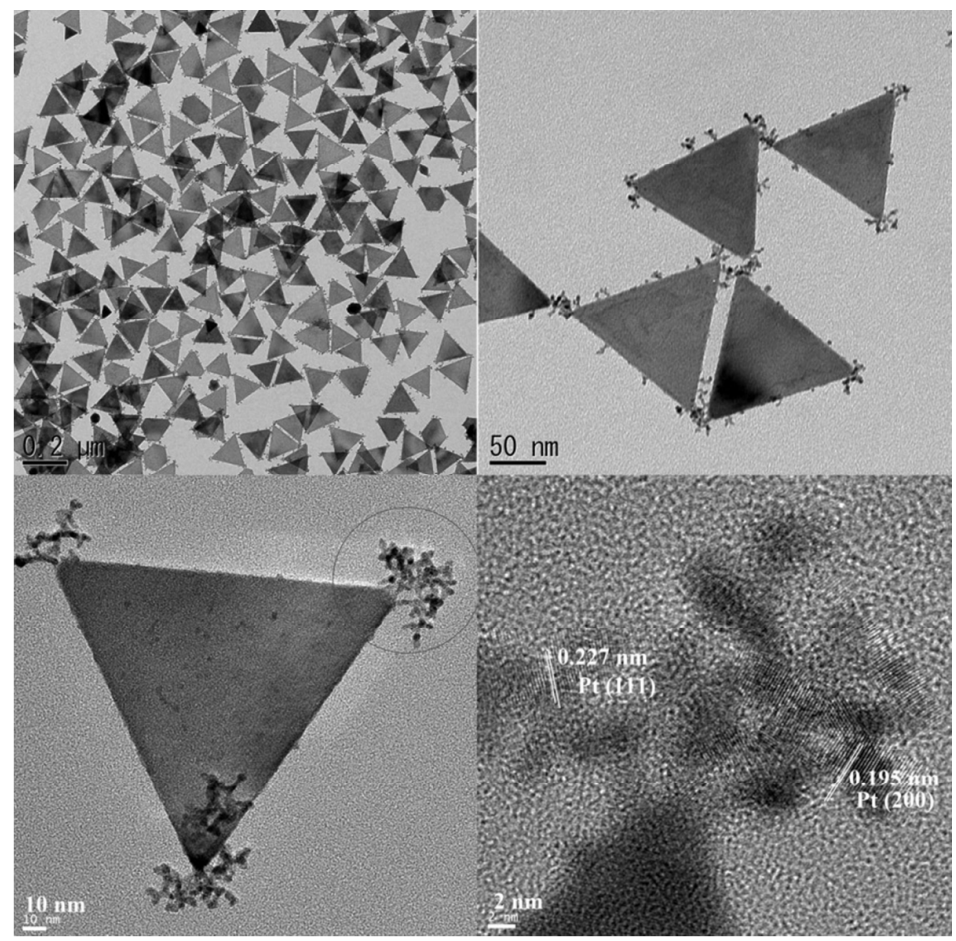

Figure S4. TEM images of Pt-tipped Au-TNPs. 

$\mathrm{KI}(10 \mathrm{mM}), 0 \mu \mathrm{L}$
$\mathrm{KI}(10 \mathrm{mM}), 10 \mu \mathrm{L}$
$\mathrm{KI}(10 \mathrm{mM}), 20 \mu \mathrm{L}$

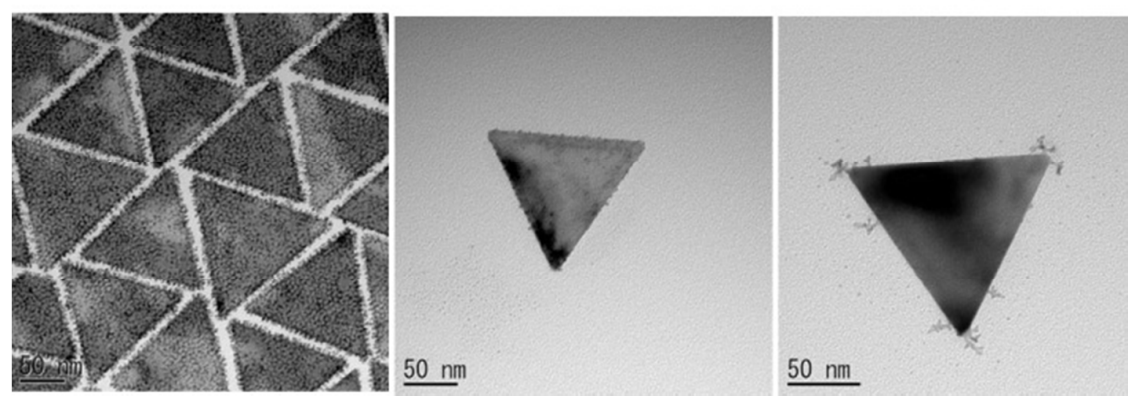

Figure S5. TEM images of Pt-loaded Au TNPs with addition of various volumes of KI solution.

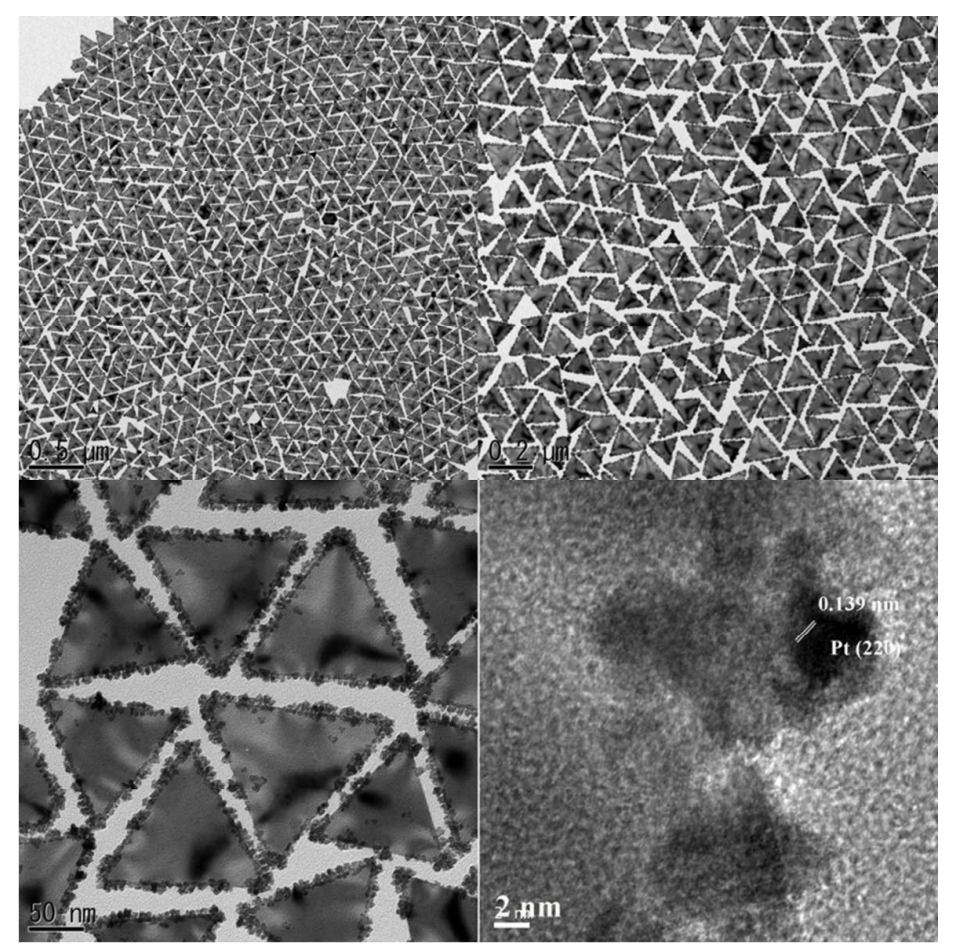

Figure S6. TEM images of Pt-edged Au-TNPs. 


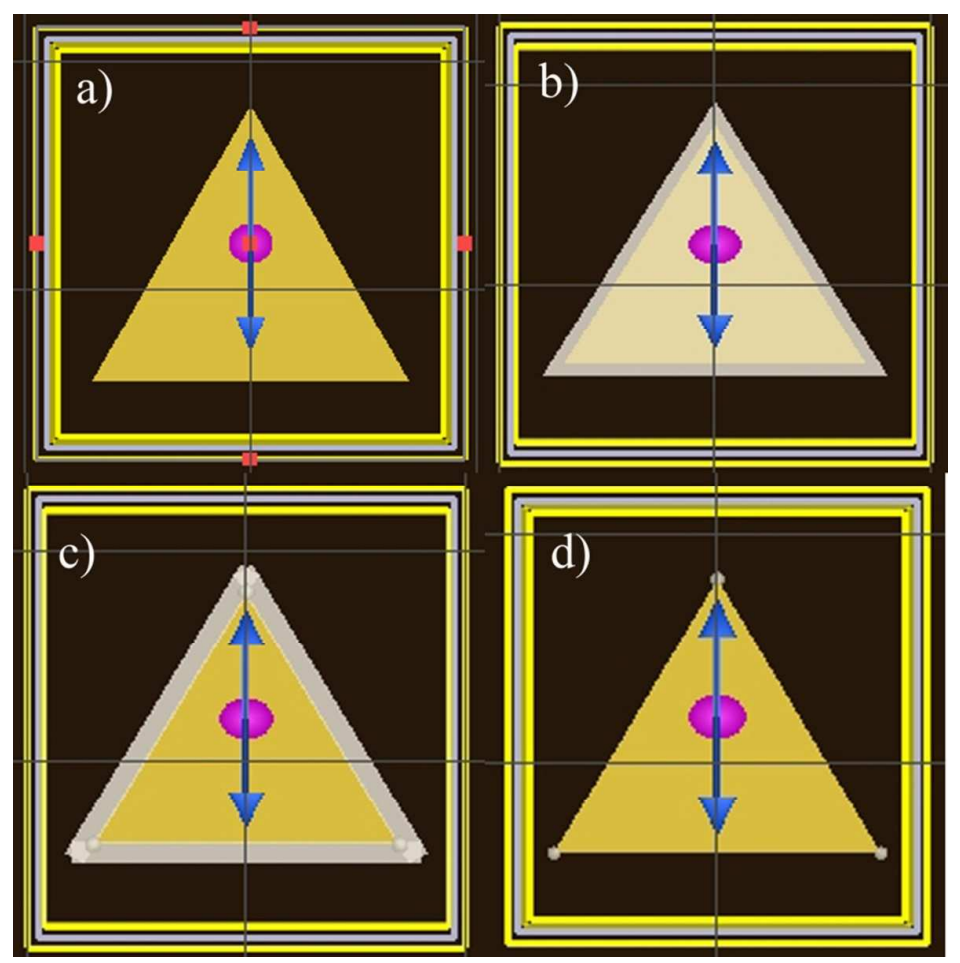

Figure S7. The constructed models for (a) Au TNPs, (b) Pt-covered Au TNPs, (c) Pt-edged Au TNPs and (d) Pt-tipped Au TNPs, respectively.

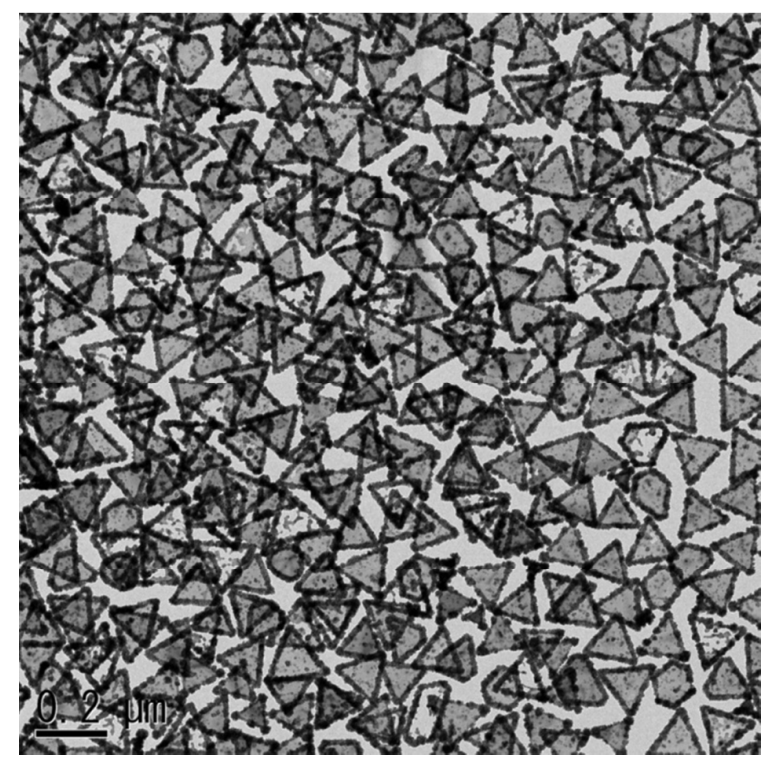

Figure S8. TEM image of Pt-edged Au TNPs with atomic 54.7 \% Pt loaded. 


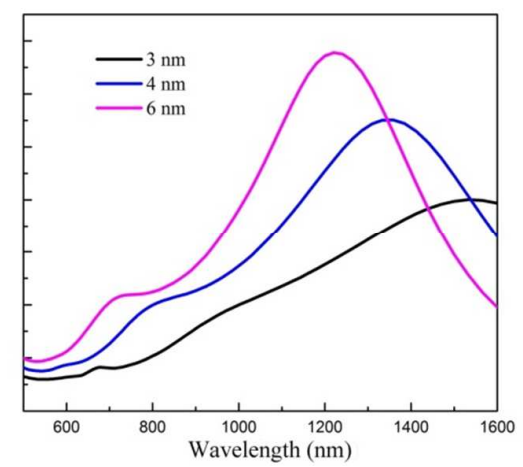

Figure S9. Calculated extinction spectra of Pt-edged Au-TNPs with various thicknesses of Au TNPs

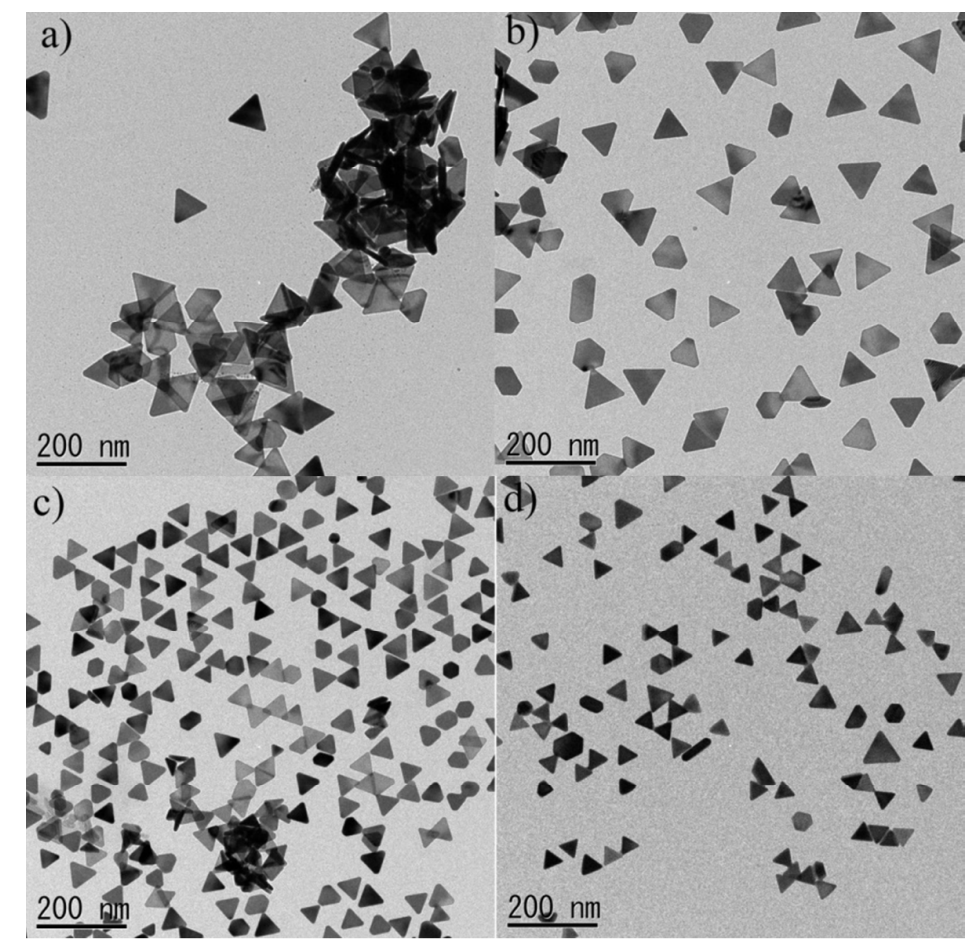

Figure S10. TEM images of Au TNPs with various sizes synthesized by addition of various seed volumes: (a) 400, (b) 600, (c) 800 and (d) $1000 \mu \mathrm{L}$. The sample a to $\mathrm{d}$ was labeled to be Au-TNPs-400, Au-TNPs-600, Au-TNPs-800 and Au-TNPs-1000, respectively. Scale bar: $200 \mathrm{~nm}$. 

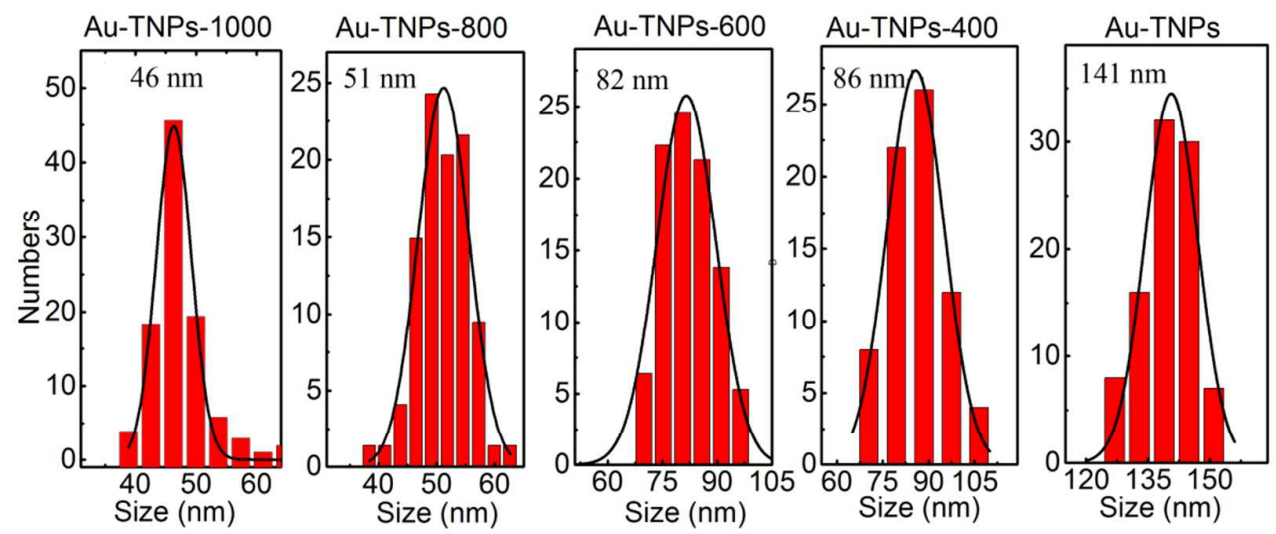

Figure S11. Size dispersion of Au TNPs with various sizes corresponding to Figure 1a and $\mathrm{S} 10$.

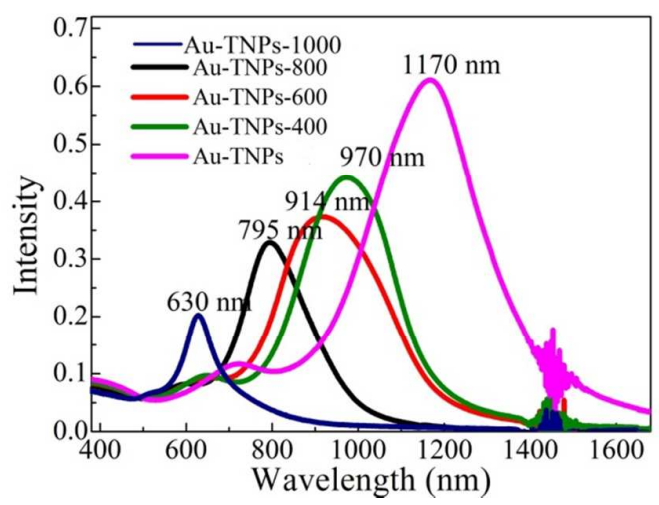

Figure S12. Visible-NIR extinction spectra of $\mathrm{Au}$ TNPs with various sizes corresponding to Figure S10.
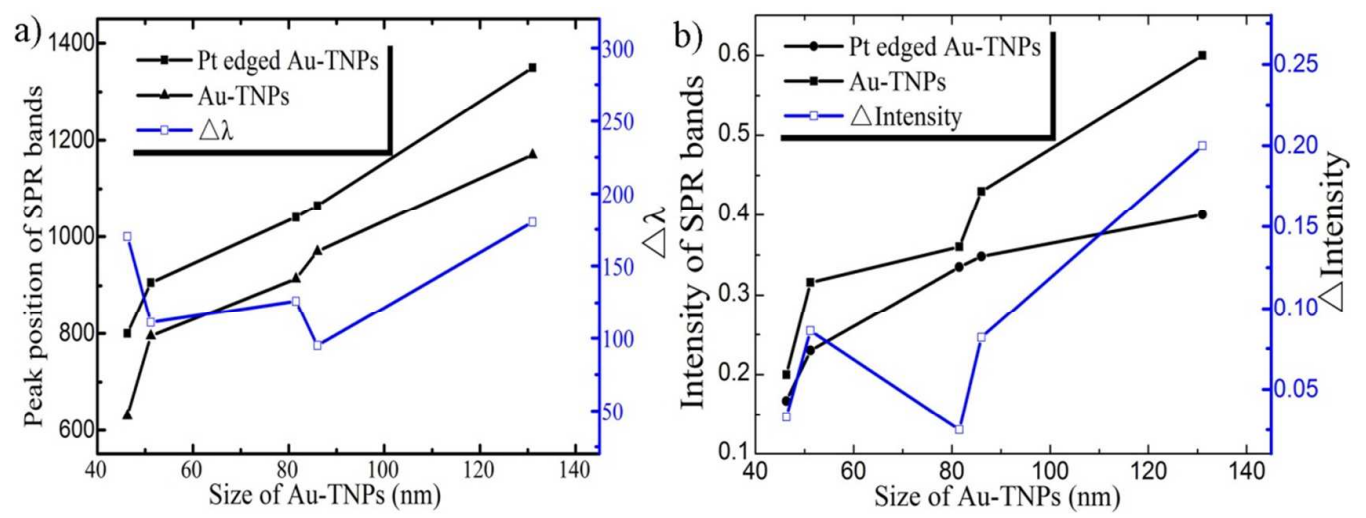

Figure S13. (a) Peak position and $\Delta \lambda$ of SPR bands of Au TNPs and Pt-edged $\mathrm{Au}$ TNPs with various sizes. (b) Intensity and $\triangle$ intensity of SPR bands of Au TNPs and Pt edged Au TNPs with various sizes. 


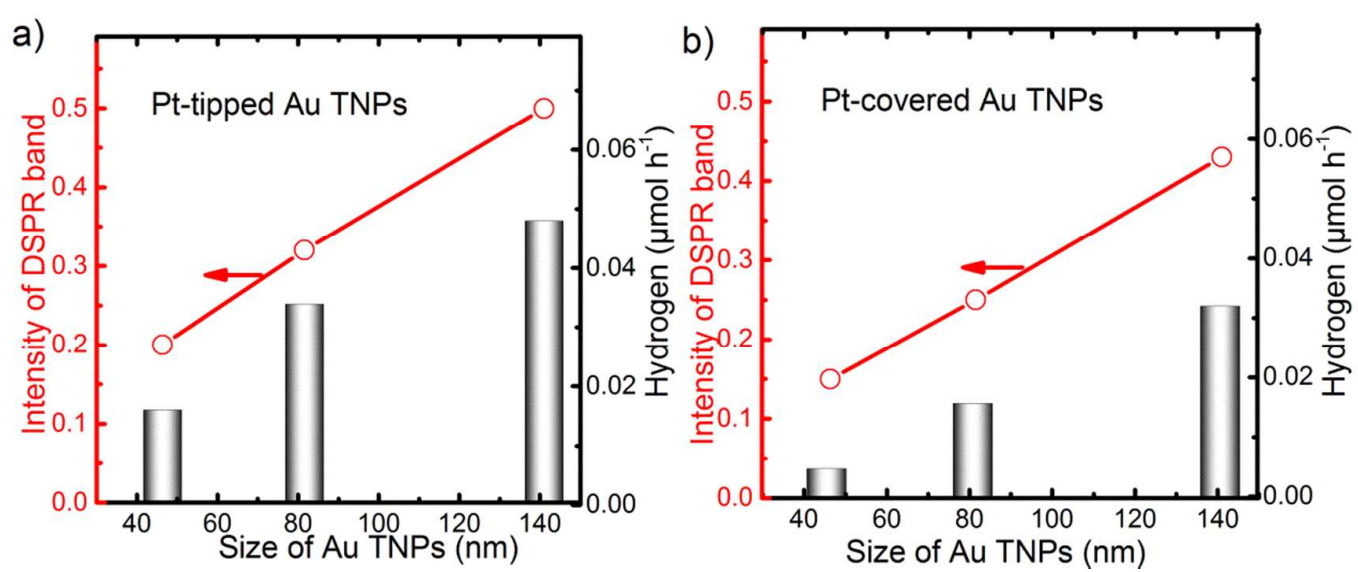

Figure S14. Intensity of DSPR band and hydrogen generation rate of Pt-tipped (a) and Pt-covered (b) Au TNPs over the size of Au TNPs.

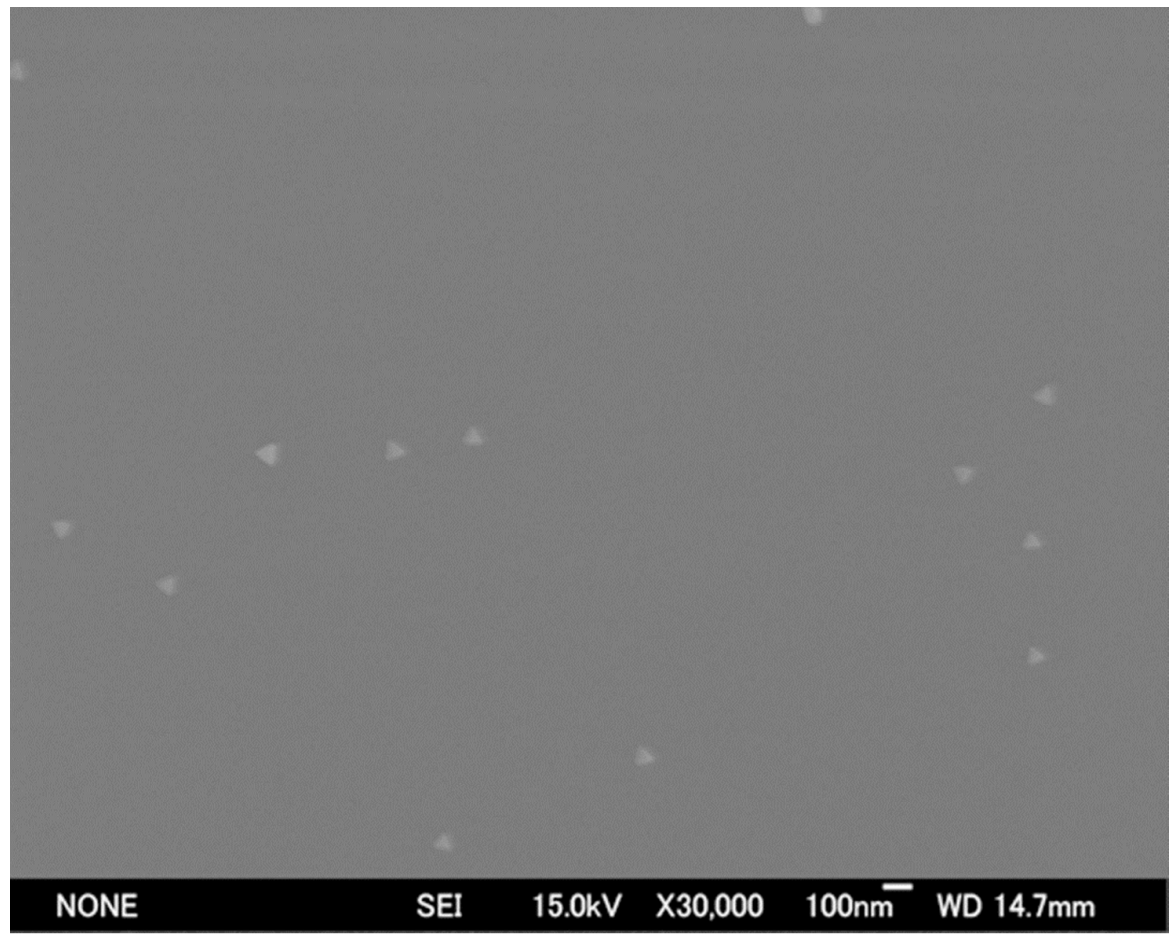

Figure S15. SEM image of Au-TNPs-600 with the size of $82 \mathrm{~nm}$ spin coated on quartz cover glass. 


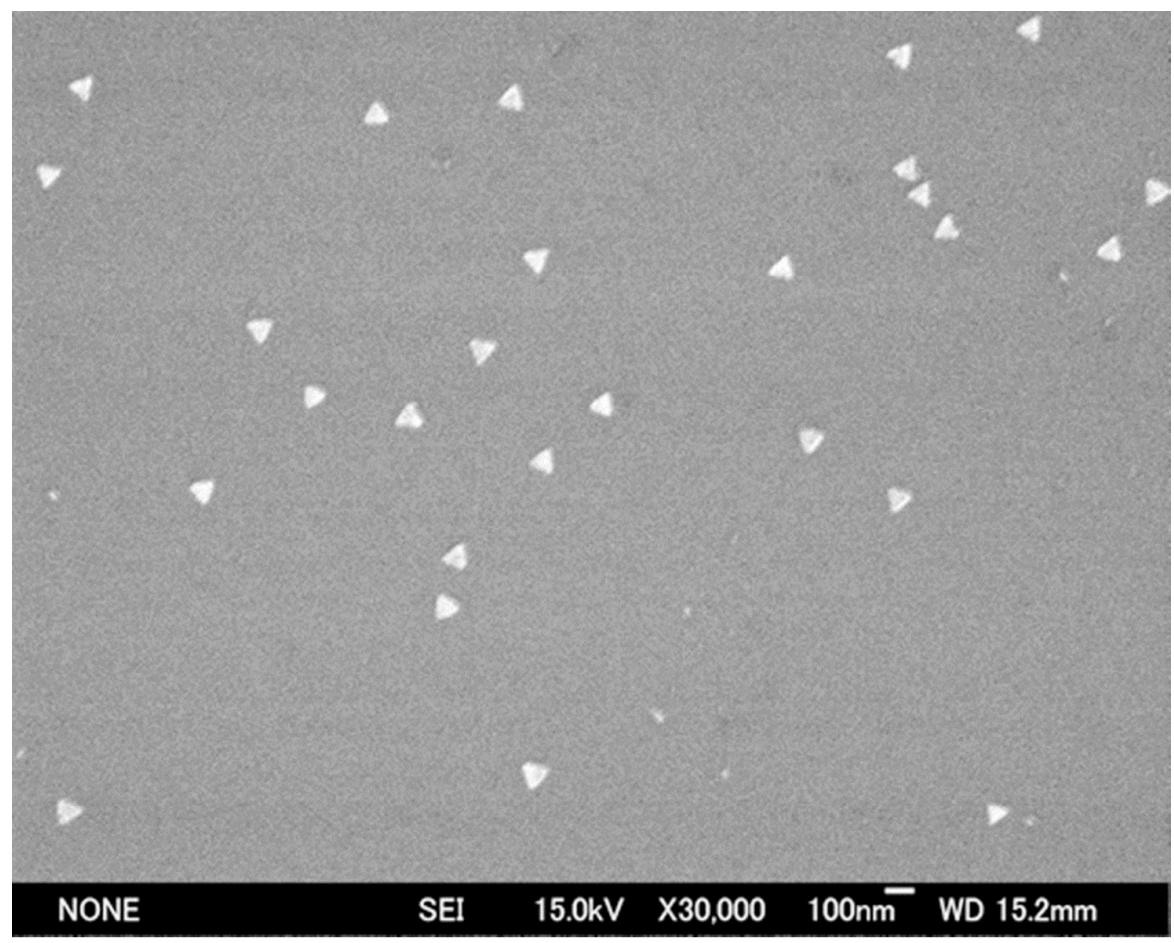

Figure S16.SEM image of Pt-edged Au-TNPs-600 spin coated on quartz cover glass.

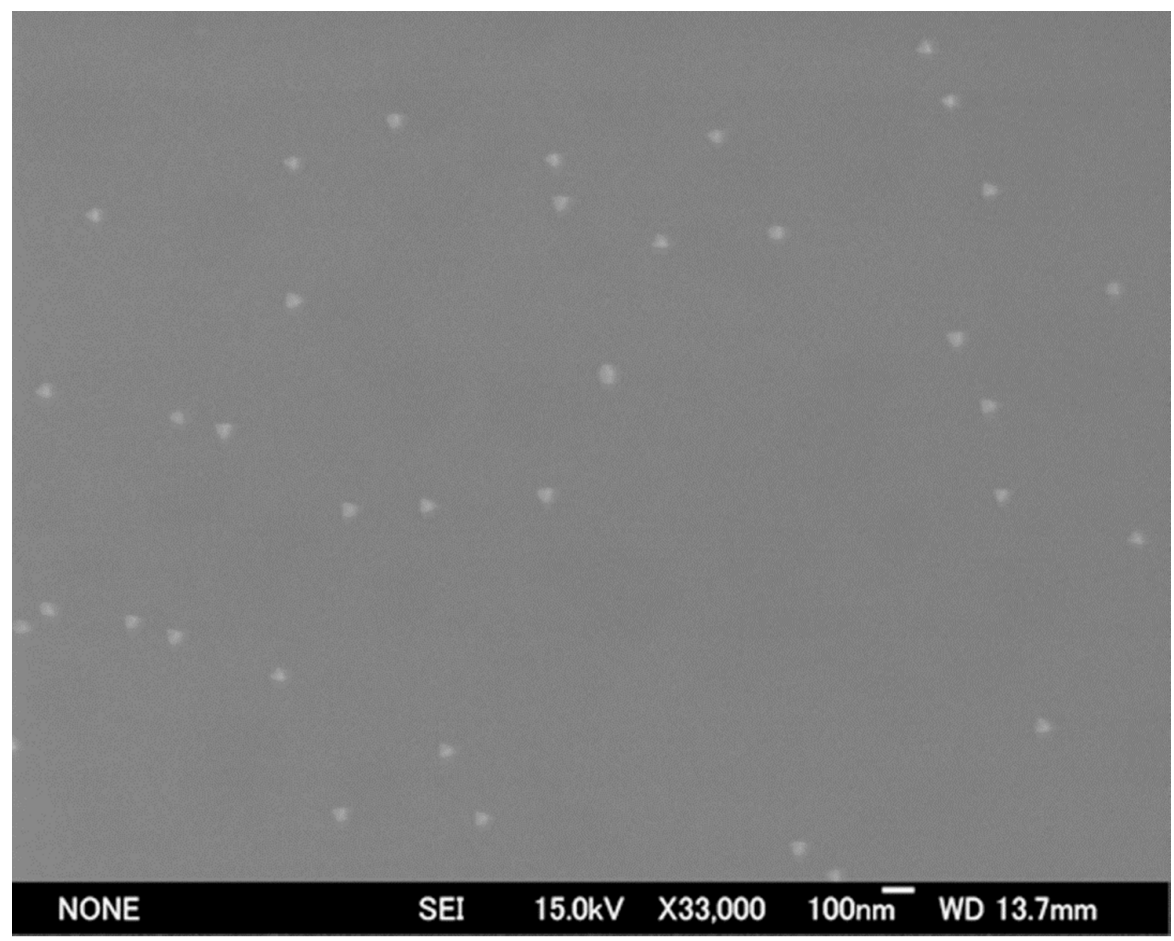

Figure S17.SEM image of Au-TNPs-800 with the size of $51 \mathrm{~nm}$ spin coated on quartz cover glass. 


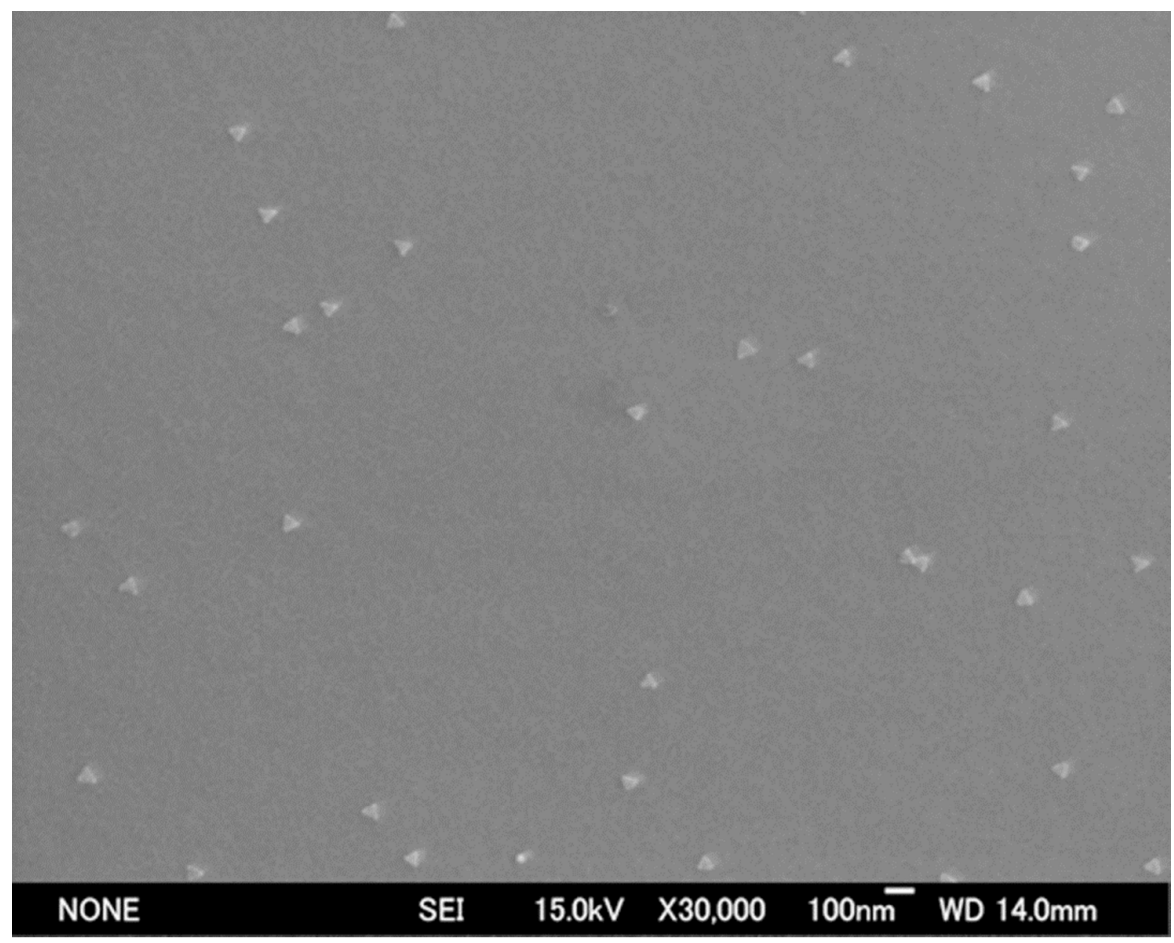

Figure S18.SEM image of Pt-edged Au-TNPs-800 spin coated on quartz cover glass.

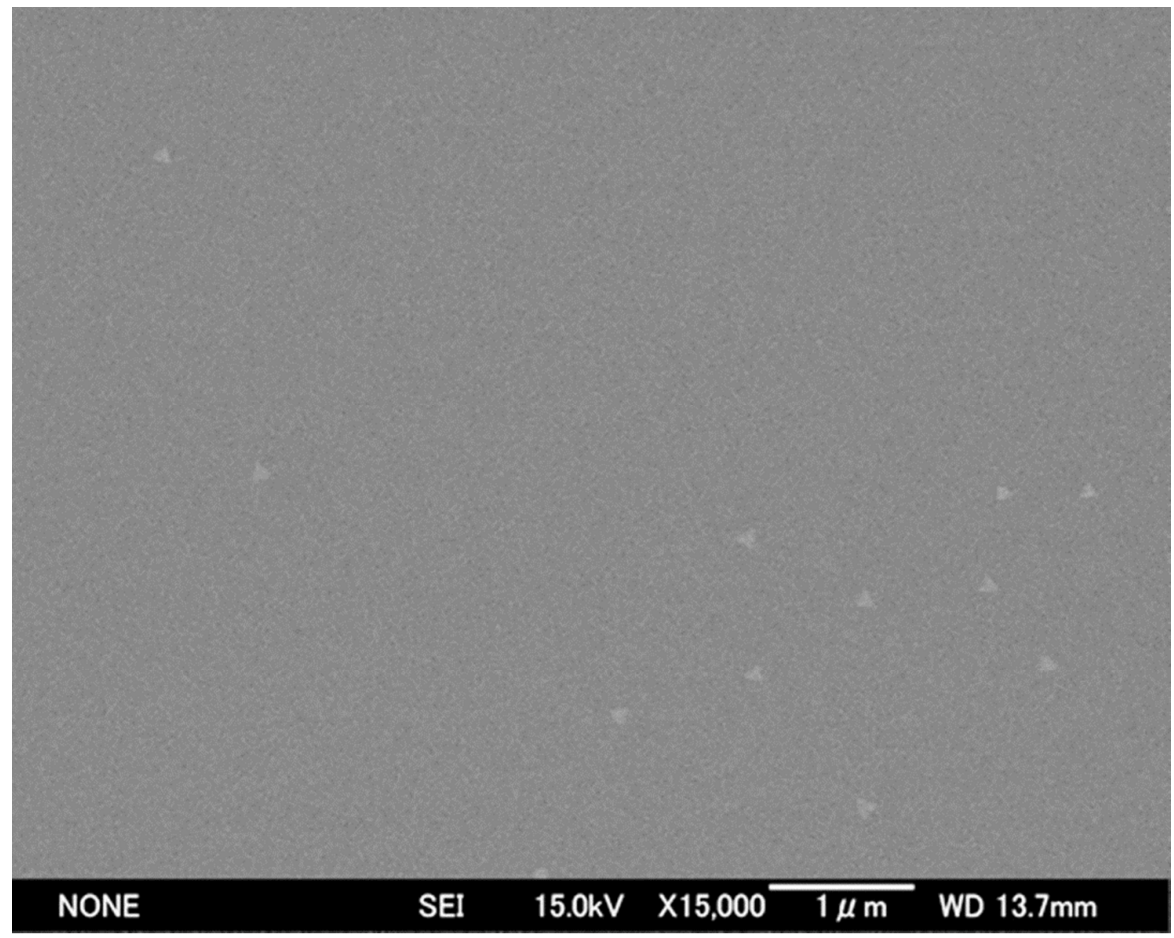

Figure S19. SEM image of Au-TNPs with the size of $141 \mathrm{~nm}$ spin coated on quartz cover glass. 


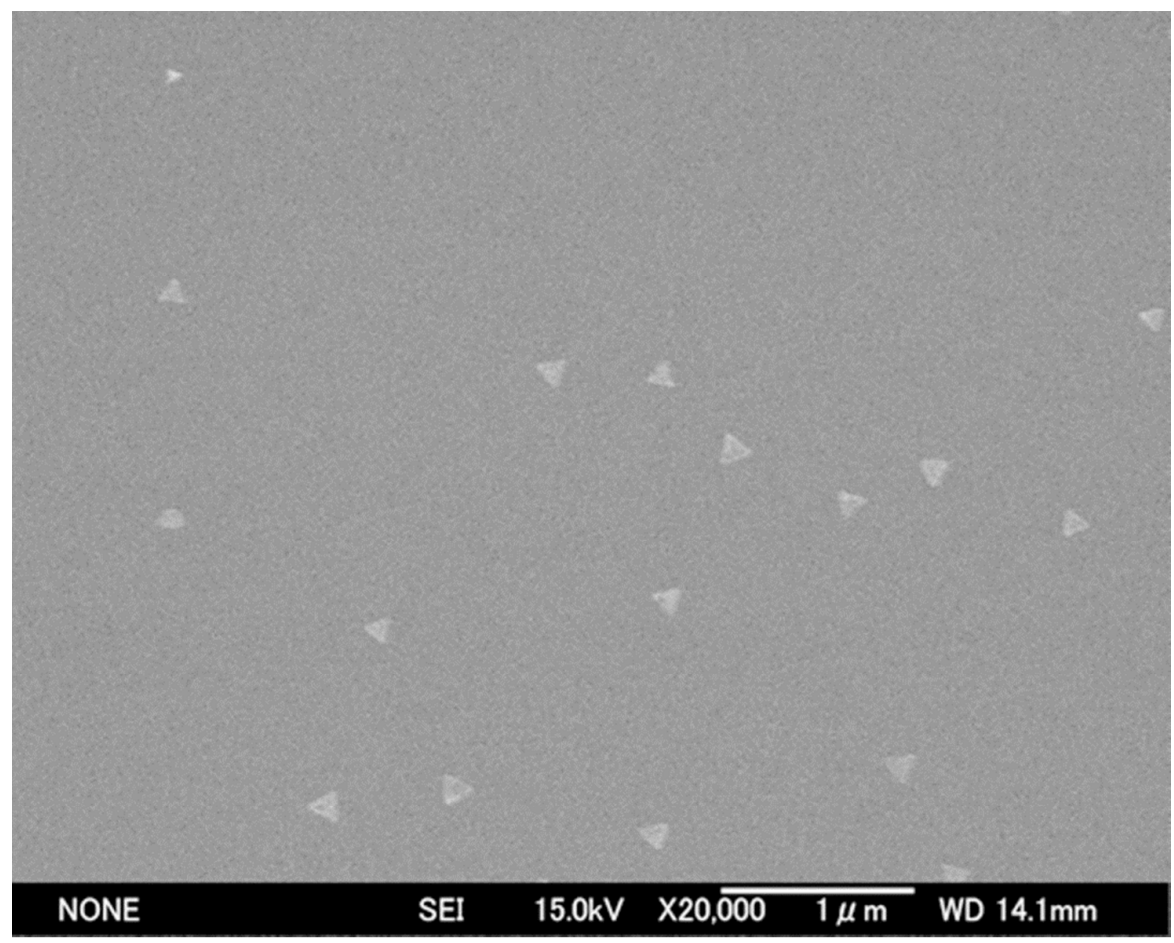

Figure S20. SEM image of Pt-edged Au-TNPs spin coated on quartz cover glass.

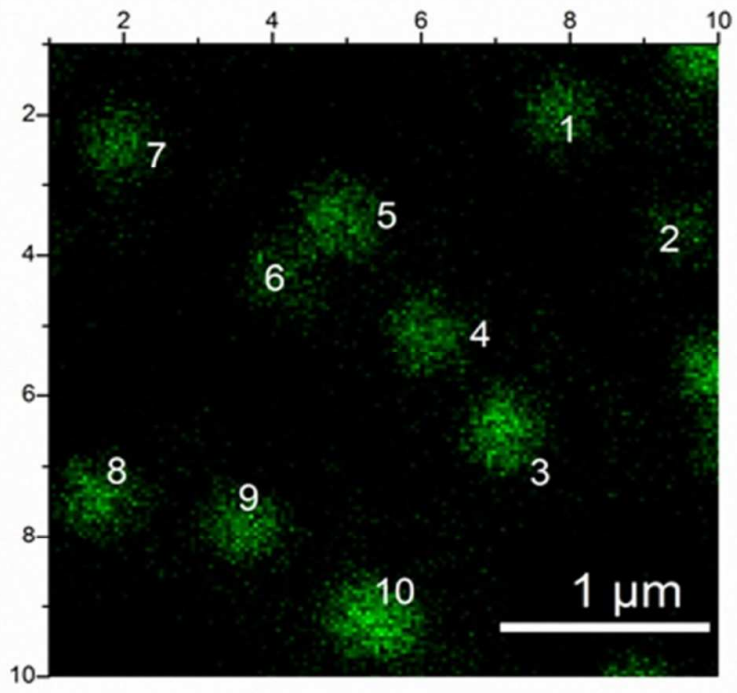

Figure S21. Single-particle PL image of Au-TNPs- 800 with the size of $51 \mathrm{~nm}$. 

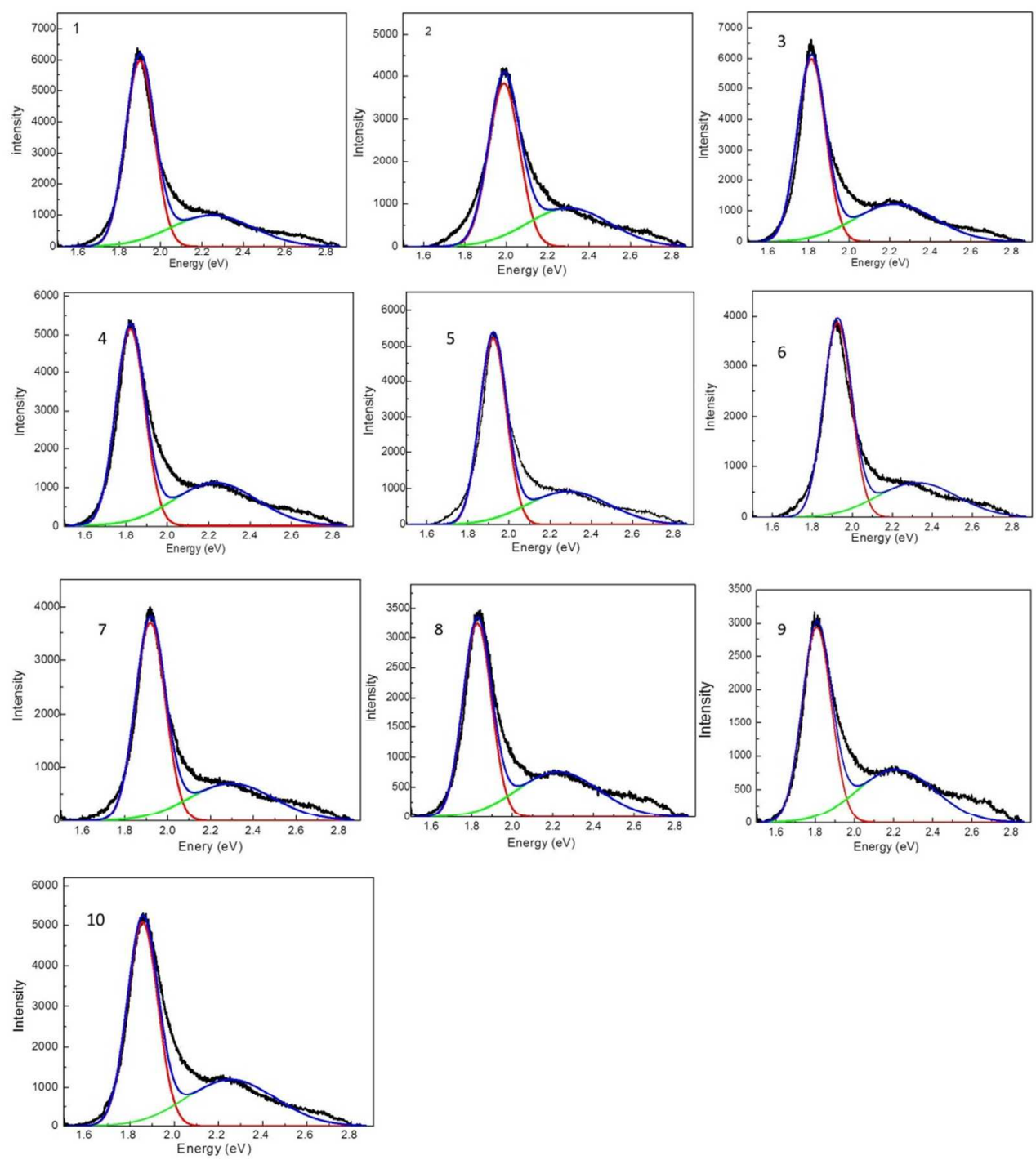

Figure S22. Single-particle PL spectra of Au-TNPs-800 corresponding to number of Figure S21. 


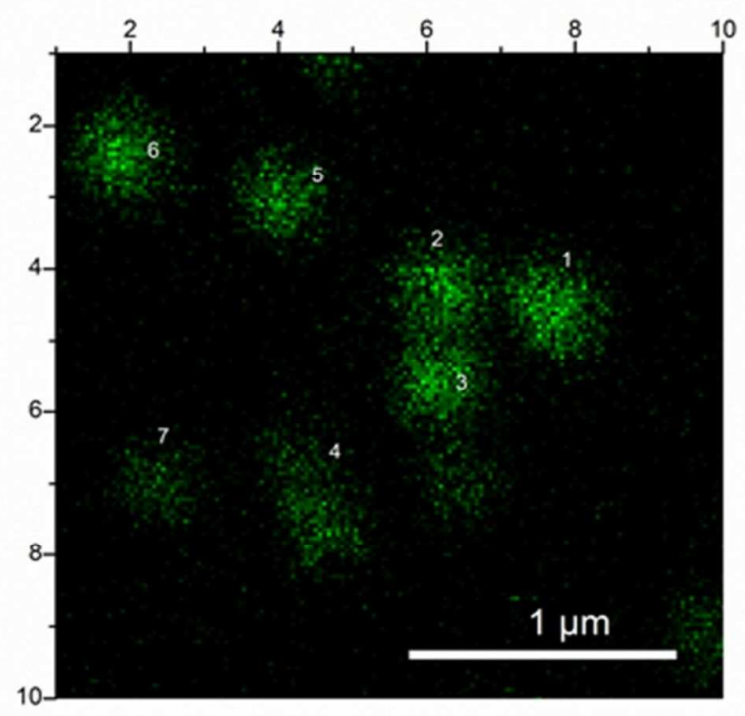

Figure S23. Single-particle PL image of Pt-edged Au-TNPs-800.
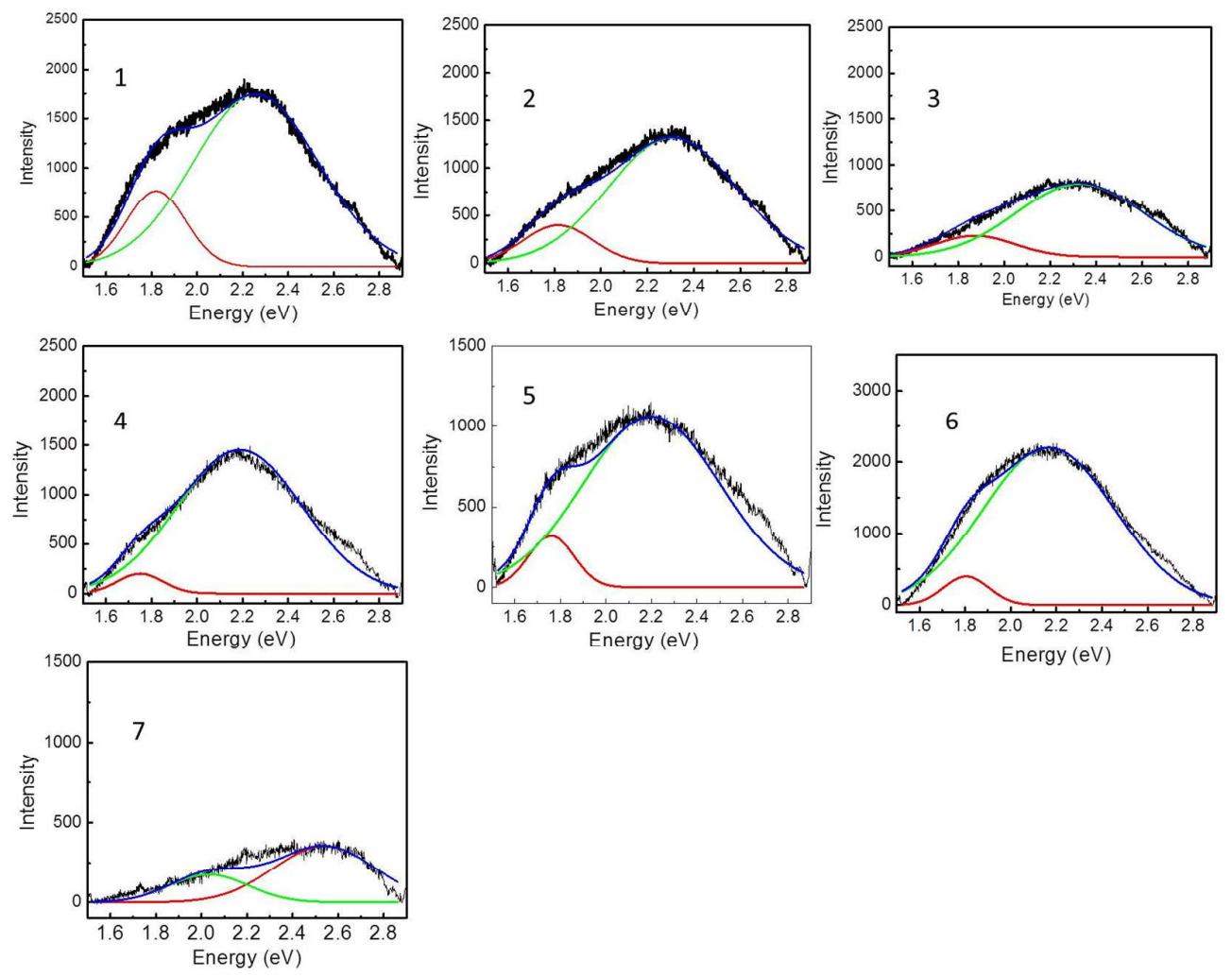

Figure S24. Single-particle PL spectra of Pt-edged Au-TNPs-800 corresponding to numbers of Figure S23. 


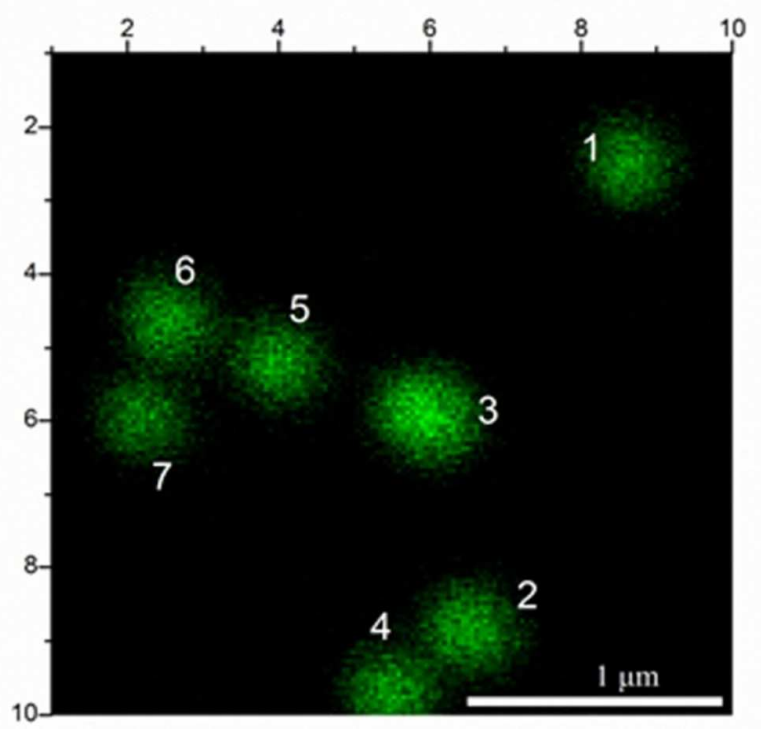

Figure S25. Single-particle PL image of Pt-edged Au-TNPs-600 with the size of 82 nm.
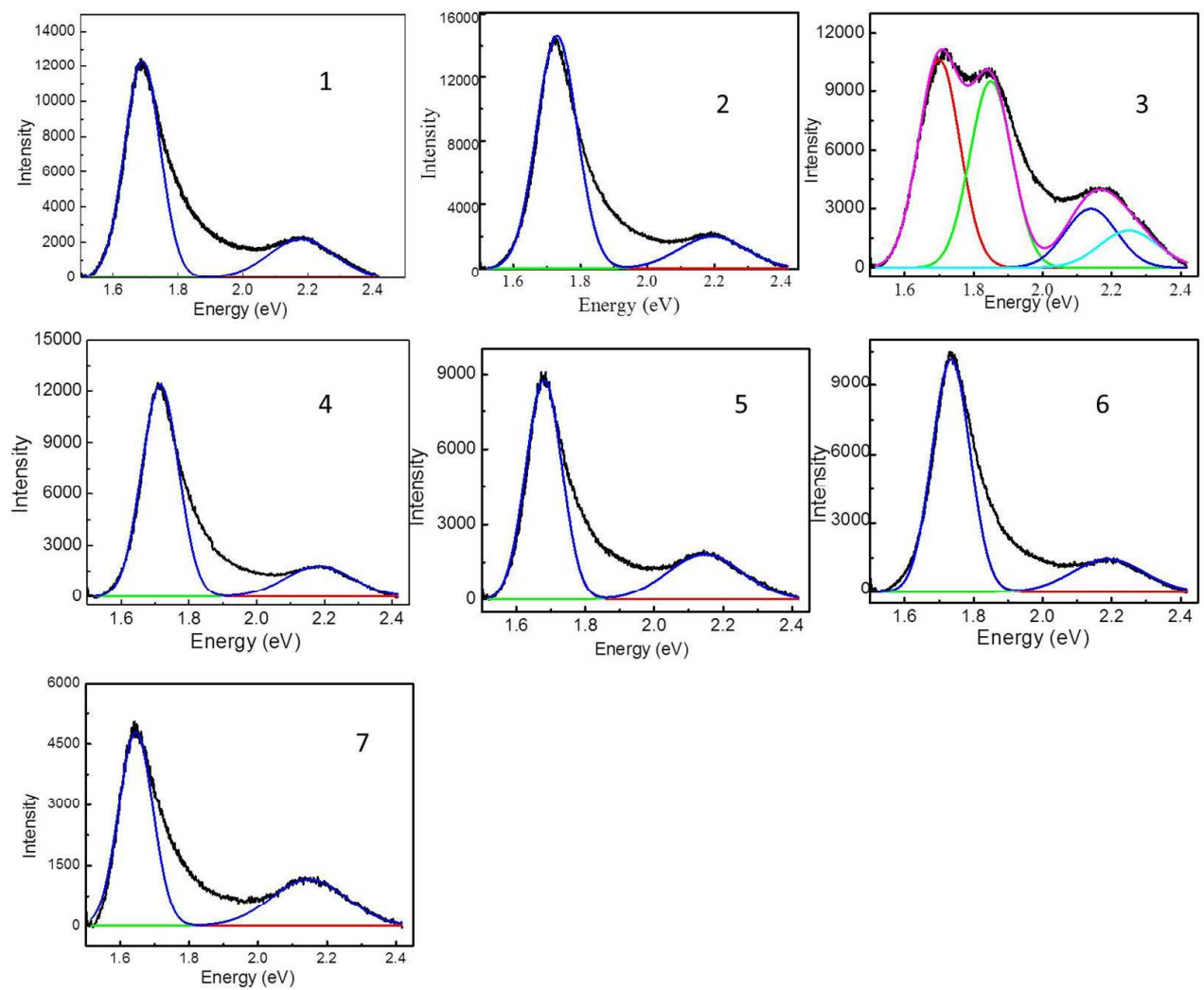

Figure S26. Single-particle PL spectra of Au-TNPs-600 corresponding to numbers of Figure S25. 


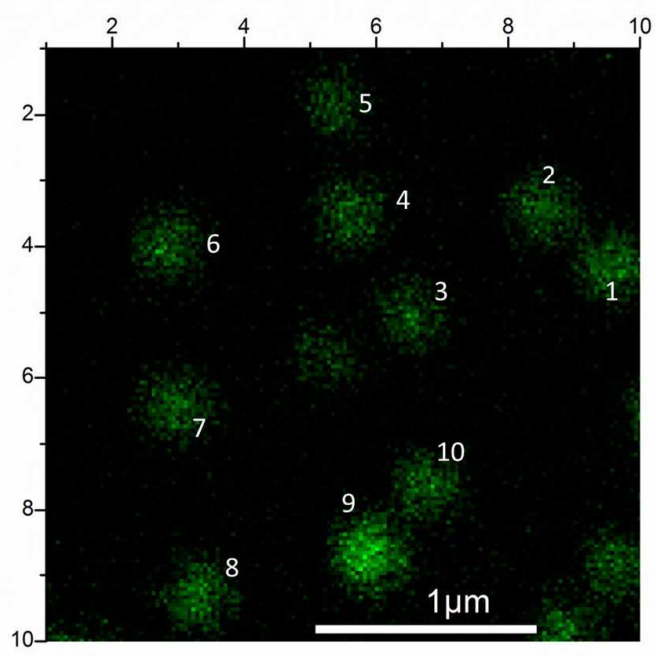

Figure S27. Single-particle PL image of Pt-edged Au-TNPs-600.
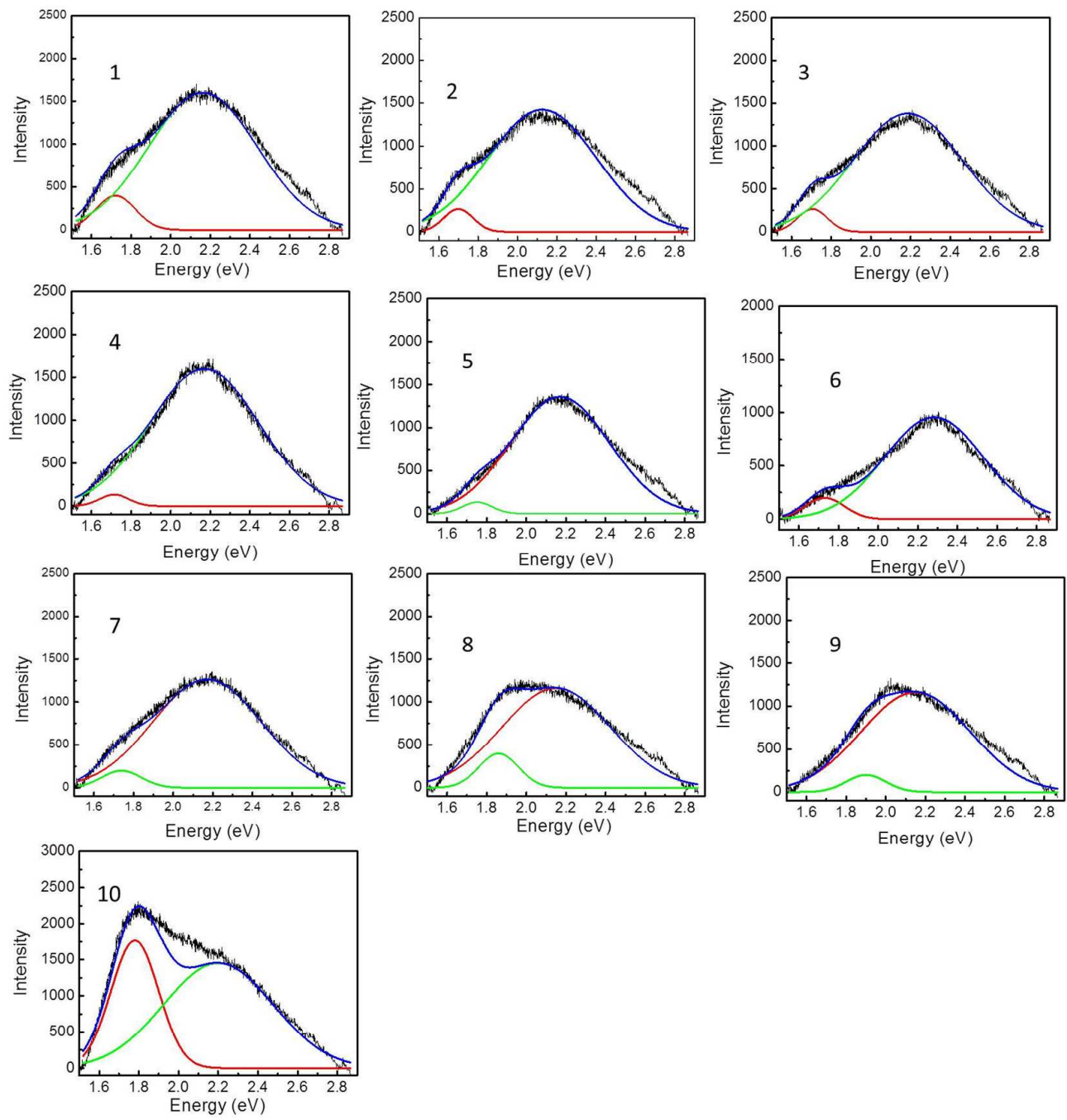
Figure S28. Single-particle PL spectra of Pt-edged Au-TNPs-600 corresponding to numbers of Figure S27.

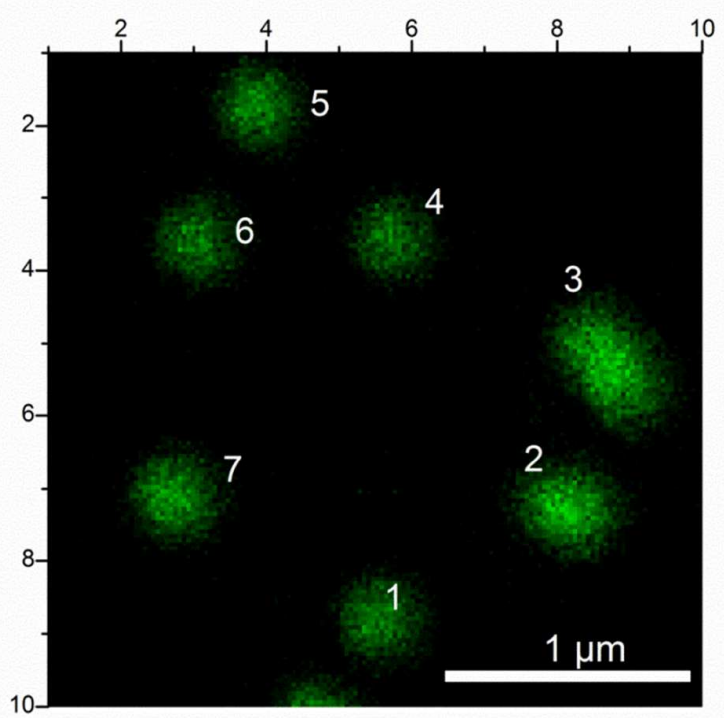

Figure S29. Single-particle PL image of Au-TNPs with the size of $141 \mathrm{~nm}$.
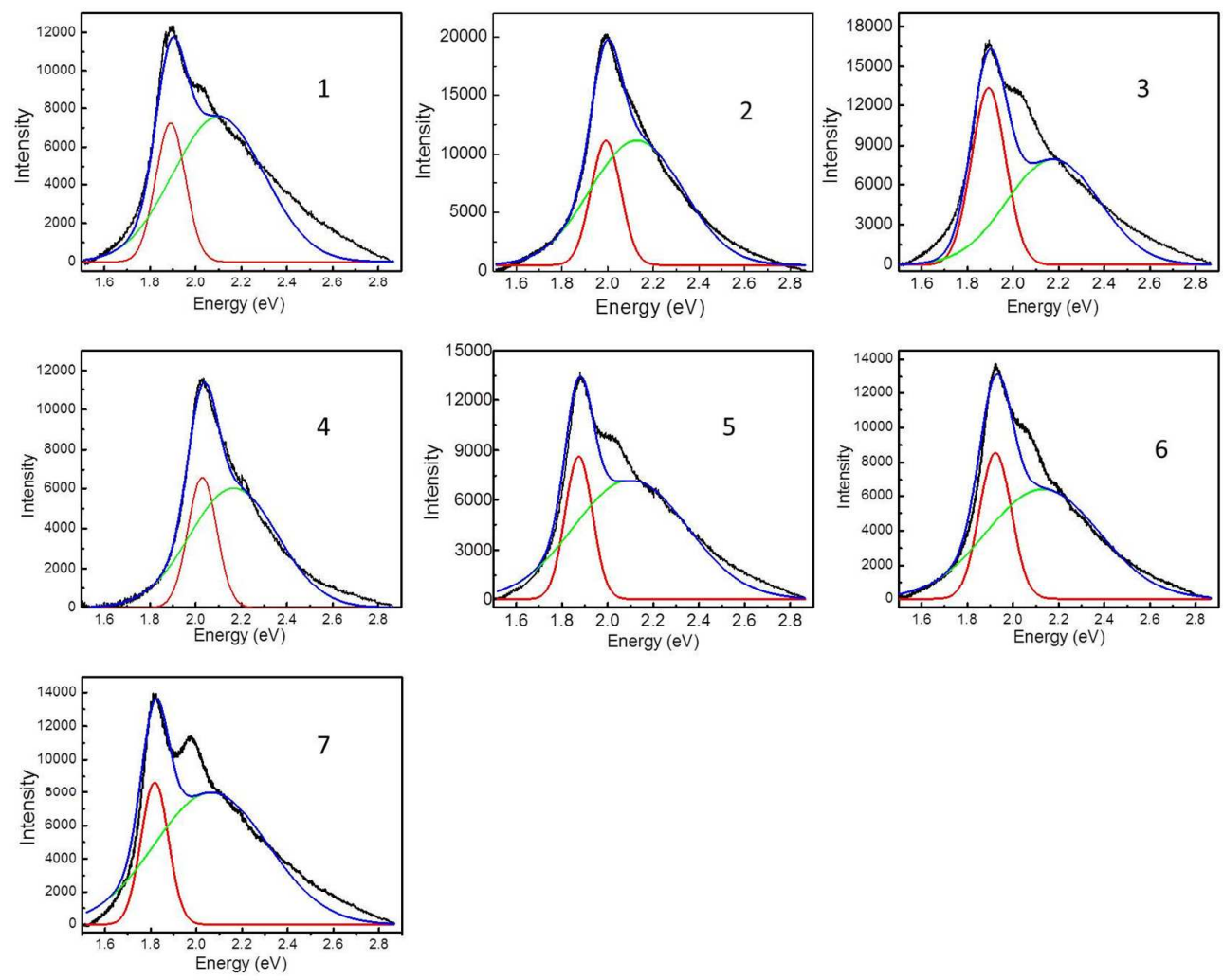

Figure S30. Single-particle PL spectra of Pt-Au-TNPs corresponding to the numbers of Figure S29. 


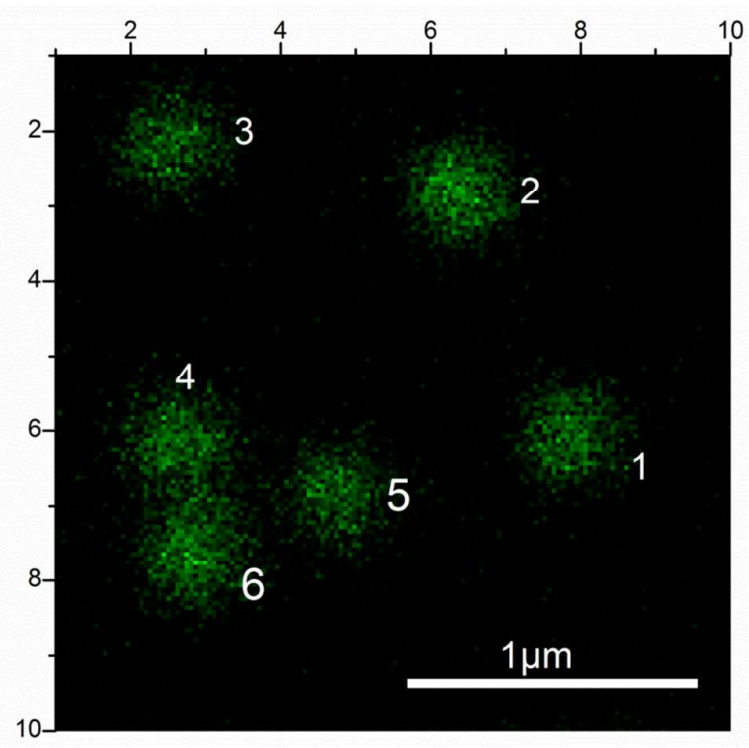

Figure S31. Single-particle PL image of Pt-edged Au-TNPs.
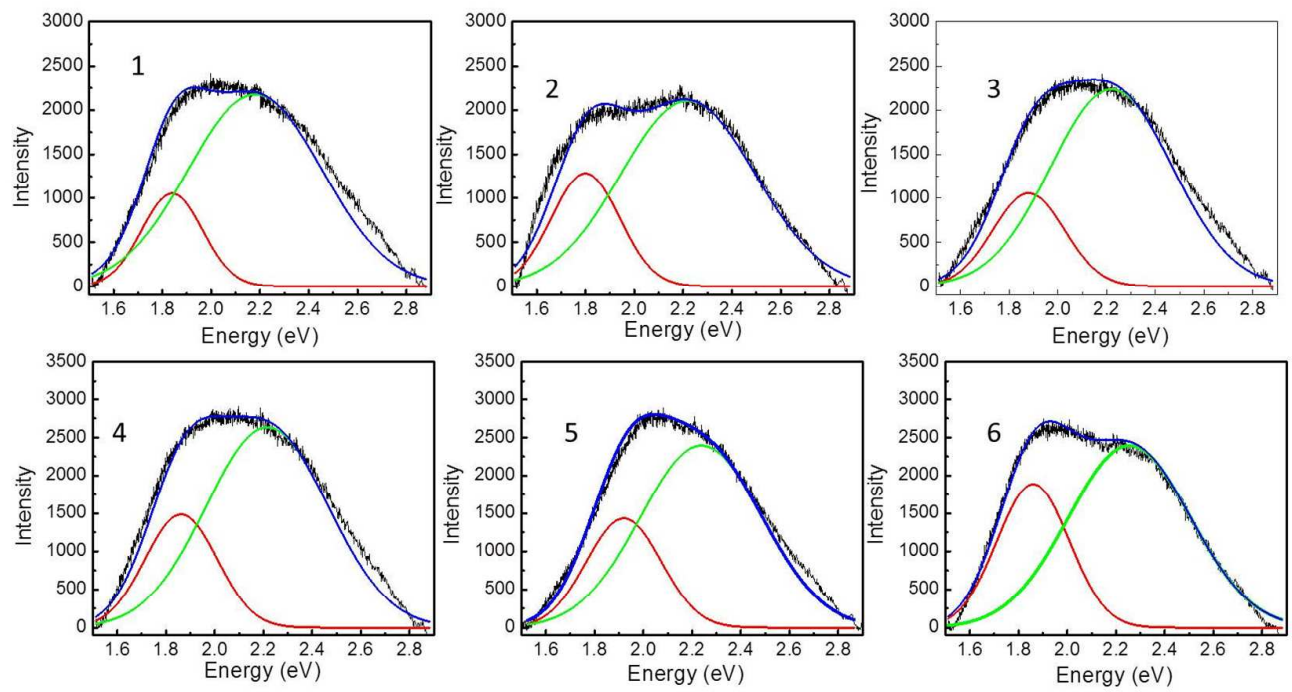

Figure S32. Single-particle PL spectra of Pt-Au-TNPs corresponding to numbers of Figure S31. 


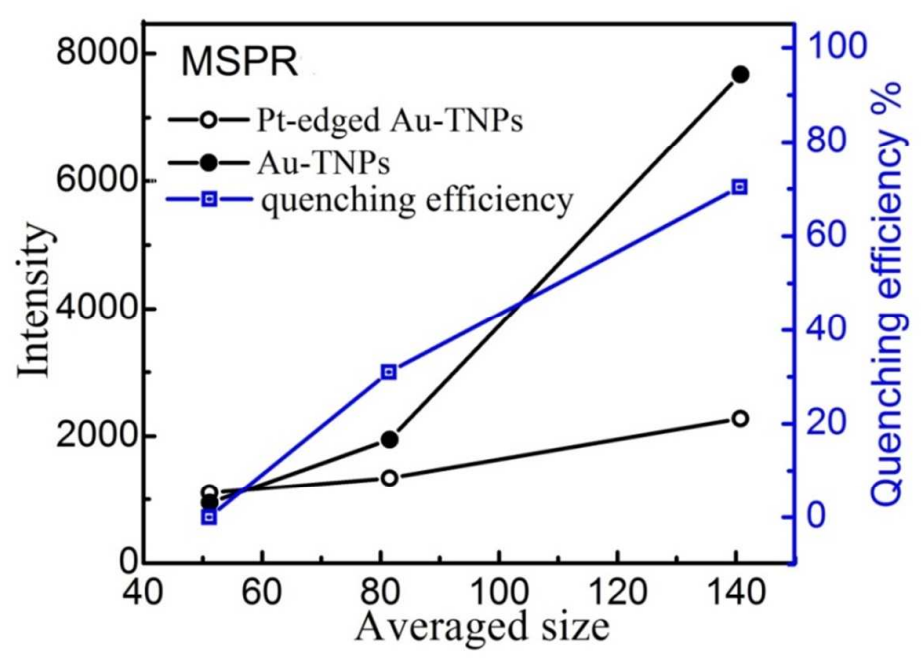

Figure S33. Intensity and quenching efficiency of the MSPR PL of Au TNPs and Pt-edged Au TNPs with the size of 141, 81 and $51 \mathrm{~nm}$.
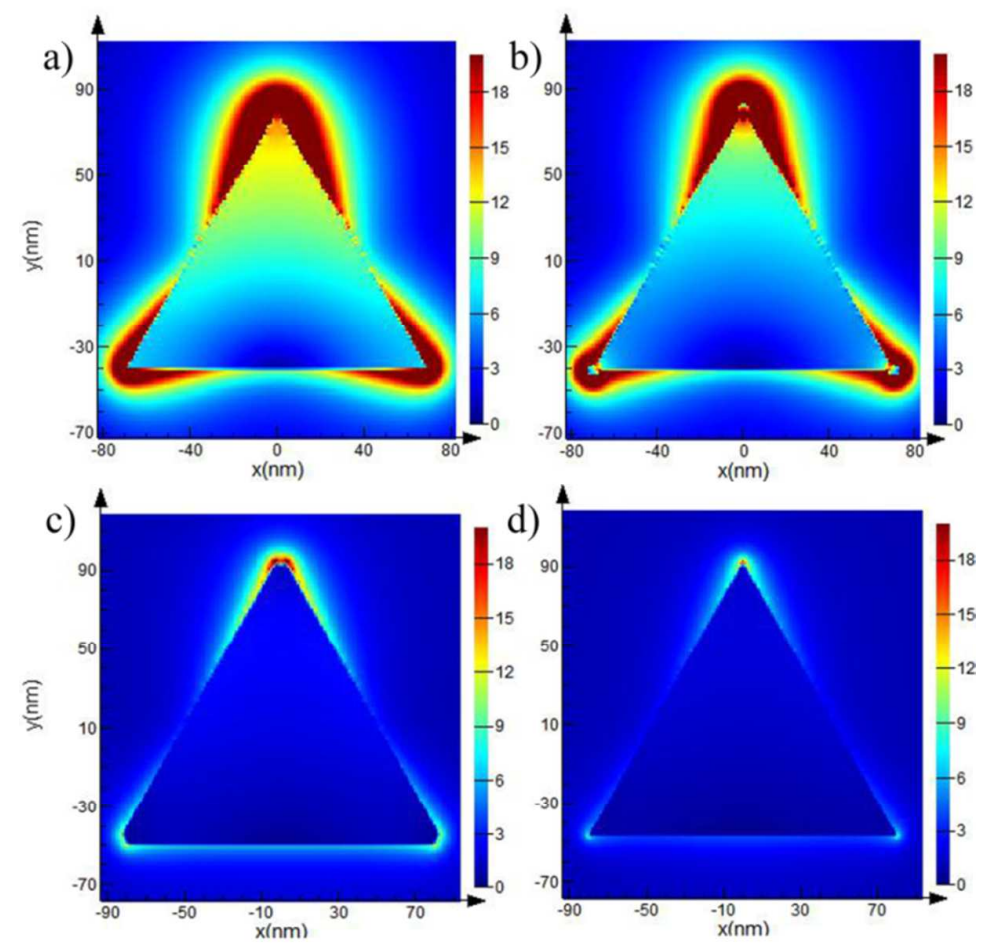

Figure S34. Electric field distribution of (a) Au TNPs, (b) Pt-tipped Au TNPs, (c) Pt-edged Au TNPs and (d) Pt-covered Au TNPs with the incident light of maximum bands.

Plasmon resonance of free-electrons can generate the strong electric field on the surface of Au-TNPs, which can promote transition of photo-generated carriers. We simulated the electric field of Au TNPs, Pt-tipped Au TNPs, Pt-edged Au TNPs and Pt-covered Au TNPs by 3D FDTD as shown in Figure S34. For pure Au TNPs, strong 
electric filed exists around the corners of TNPs.(Figure S34a) When Pt NPs are tipped on Au-TNPs, the tip still has a strong electric field (Figure S34b) which can promote the transfer of electrons from Au to Pt. However, corner area of Au-TNPs is small and limits the amount of Pt loaded, which adverse to the efficient transfer of electrons. For Pt-edged Au TNPs, the electric field (Figure S34c) becomes weaker than that of Pt-tipped one attributing to variation of free-electrons when a large amount of Pt NPs loaded on Au TNPs. Moreover, Pt-edged Au TNPs has more boundaries near the corners of Au TNPs than Pt-tipped ones, which is favor for the hot electron transfer, leading to high activity of Pt-edged Au TNPs in hydrogen evolution. When Au TNPs are covered by Pt NPs, SPR bands of Au-TNPs become weak which have been confirmed by the visible-NIR spectra and simulations. The electric fields of Pt-covered Au TNPs are also weaker than Pt-tipped and Pt-edged ones as shown in Figure S34d, which adverse the transfer of electrons. Furthermore, Pt randomly loaded on Au-TNPs will provide more recombination sites for photo-generated electron-hole. Both above two effects low the activity of Pt-covered Au-TNPs in hydrogen generation. 